\title{
Computational identification of miRNAs and their targets in Phaseolus vulgaris
}

\author{
J. Han, H. Xie, M.L. Kong, Q.P. Sun, R.Z. Li and J.B. Pan \\ Beijing Key Laboratory for Agricultural Application and New Technique, \\ College of Plant Science and Technology, Beijing University of Agriculture, \\ Beijing, China
}

Corresponding authors: J. Han / J.B. Pan

E-mail: hanjun422@yahoo.com.cn / bacpjb@yahoo.com.cn

Genet. Mol. Res. 13 (1): 310-322 (2014)

Received April 8, 2013

Accepted September 5, 2013

Published January 17, 2014

DOI http://dx.doi.org/10.4238/2014.January.17.16

\begin{abstract}
MicroRNAs (miRNAs) are a class of non-coding small RNAs that negatively regulate gene expression at the posttranscriptional level. Although thousands of miRNAs have been identified in plants, limited information is available about miRNAs in Phaseolus vulgaris, despite it being an important food legume worldwide. The high conservation of plant miRNAs enables the identification of new miRNAs in P. vulgaris by homology analysis. Here, 1804 known and unique plant miRNAs from 37 plant species were blast-searched against expressed sequence tag and genomic survey sequence databases to identify novel miRNAs in P. vulgaris. All candidate sequences were screened by a series of miRNA filtering criteria. Finally, we identified 27 conserved miRNAs, belonging to 24 miRNA families. When compared against known miRNAs in $P$. vulgaris, we found that 24 of the 27 miRNAs were newly discovered. Further, we identified 92 potential target genes with known functions for these novel miRNAs. Most of these target genes were predicted to be involved in plant development, signal transduction, metabolic pathways, disease resistance, and environmental stress response. The identification of the novel miRNAs in P. vulgaris is anticipated to
\end{abstract}


provide baseline information for further research about the biological functions and evolution of miRNAs in $P$. vulgaris.

Key words: Computational identification; MicroRNAs; Target genes; Phaseolus vulgaris

\section{INTRODUCTION}

MicroRNAs (miRNAs) are a newly discovered class of endogenous, highly conserved, non-coding small RNAs. Most mature miRNAs are approximately 21 nucleotides (nt) in length, with their precursor being able to fold into a stem-loop structure (Bartel, 2004). miRNAs have been broadly reported to be located on non-coding regions of genomes from various species (Megraw and Hatzigeorgiou, 2010). The MIR genes of plants are first transcribed by polymerase II to produce primary miRNA (pri-miRNA), which is then processed by the enzyme Dicer like 1 (DCL1) into a product called precursor miRNA (pre-miRNA). This precursor has a stem-loop structure, and the mature miRNA sequence is located on one arm of the hairpin structure. The pre-miRNA is subsequently cleaved into the double-stranded mature miRNA: Mirna* duplex (Bartel, 2004). Finally, the single-stranded mature miRNA is assembled into RNA-induced silencing complex (RISC), to induce it to bind to the complementary target mRNA (Bartel, 2004). Increasing evidence has shown that miRNAs have multiple roles in plant biological processes by negatively regulating their target proteins, including plant organ development and differentiation, signal transduction, morphogenesis, and adaptive responses to diverse biotic and abiotic stresses (Mallory et al., 2005; Larue et al., 2009; Li et al., 2012; Sunkar et al., 2012).

Since the first plant miRNA was identified in Arabidopsis, their crucial roles in posttranscriptional gene regulation have received increasing attention. Tremendous effort has been made to identify new miRNAs in different plant species, resulting in the discovery of large numbers of miRNAs in plants. At present, approximately 5000 mature miRNAs have been discovered from plants, and deposited in the public available miRNA database (miRbase, Release August 19, 2012). Most of these miRNAs were identified from plants with sequenced genomes, including 675 from Medicago truncatula, 591 from Oryza sativa, 506 from Glycine max, 323 from Populus trichocarpa, 299 from Arabidopsis thaliana, 206 from Sorghum bicolor, 201 from Arabidopsis lyrata, 174 from Zea mays, 163 from Vitis vinifera, and 142 from Brachypodium distachyon. To date, only 8 miRNAs have been identified from Phaseolus vulgaris, and deposited in the current edition of the miRNA registry.

The common bean (P. vulgaris L.) is one of the most important food legumes worldwide, and it has been suggested that it should be used as a model species for studying legume crops (Broughton et al., 2003). Although its genome sequence is unknown, the public EST and GSS databases make it possible to identify miRNAs in $P$. vulgaris by comparative genomebased homology searching. In this study, 1804 known and unique plant miRNA sequences were employed to search the EST and GSS databases to predict the potential miRNAs in $P$. vulgaris. A total of 27 potential miRNAs were detected, 24 of which were newly discovered in P. vulgaris. Subsequently, we also blasted these newly predicted miRNA sequences against the $P$. vulgaris mRNA database. We found that 92 potential target genes with known functions were predicted for these newly identified miRNAs. This study is anticipated to facilitate re- 
search focused on elucidating the regulatory roles of miRNAs in plant growth, organ development, metabolism, and other biological processes in $P$. vulgaris.

\section{MATERIAL AND METHODS}

\section{Mature miRNA query sequences, Phaseolus vulgaris EST, and GSS}

A total of 3580 previously known plant mature miRNA sequences from 37 plant species were downloaded from miRbase (http://www.mirbase.org/). After the removal of redundant sequences, the remaining unique miRNA sequences were employed as query sequences to blast against the Phaseolus vulgaris EST and GSS database, which were available online from the National Center for Biotechnology Information (NCBI) GenBank nucleotide databases (http://www.ncbi.nlm.nih.gov/).

\section{Availability of software}

BLASTN and BLASTX (http://www.ncbi.nlm.nih.gov/BLAST/) was used to search for $P$. vulgaris miRNA homologs and to analyze the protein-encoding sequences, respectively. Based on the ClustalW alignments by Codoncode Aligner, the repeated EST or GSS of the same genes were discarded. The Zuker RNA folding algorithm software MFold 3.5 (Zuker, 2003), which is freely available online (http://mfold.rna.albany.edu/?q=mfold), was used to generate the secondary structures of the RNAs. Target genes for the novel miRNAs were predicted by the web tool psRNATarget (Dai and Zhao, 2011) (http://bioinfo3.noble.org/ psRNATarget/).

\section{Procedure and screening criteria for miRNA identification}

The procedure for the identification of potential miRNAs was shown in Figure 1. Previously known plant miRNAs were screened to remove redundant sequences, and the remaining unique miRNA sequences were used as queries to search for miRNA homologs in $P$. vulgaris. Searching parameters were set as follows: maximum target sequences for 1000 , expect threshold for 10, the remaining parameters were default. All of the EST or GSS, with no more than 4 mismatches against the query sequences, were saved. The repeated sequences of the same genes were discarded by Codoncode Aligner. The remaining sequences were then used to conduct a BLASTX analysis to remove the protein-encoding sequences.

The stem-loop structures of candidate precursor miRNAs were generated by the software MFold 3.5 (Zuker, 2003), where the default parameters were used. The following criteria were employed to screen the potential $P$. vulgaris miRNAs or pre-miRNAs: 1) predicted mature miRNAs should have no more than 4 nucleotide mismatches with the known plant miRNAs, 2) pre-miRNA sequence within an EST or GSS could fold into a perfect or near perfect stem-loop hairpin secondary structure, 3) the mature miRNA sequence should be located on one arm of the hairpin structure, 4) no more than 6 mismatches between the potential mature miRNA sequence and the opposite miRNA* sequence were allowed, 5) no loops or breaks in miRNA sequences were allowed, 6) the secondary structures of predicted pre-miRNA should have higher MFEI (minimal folding-free energy index) and negative MFE 
(minimal free energy) compared to other small RNAs, 7) MFE should be lower than -20 kcal/ mol, while MFEI should be over 0.85 , and 8 ) the content of $\mathrm{A}+\mathrm{U}$ should be within 30 to $70 \%$ (Ambros et al., 2003).

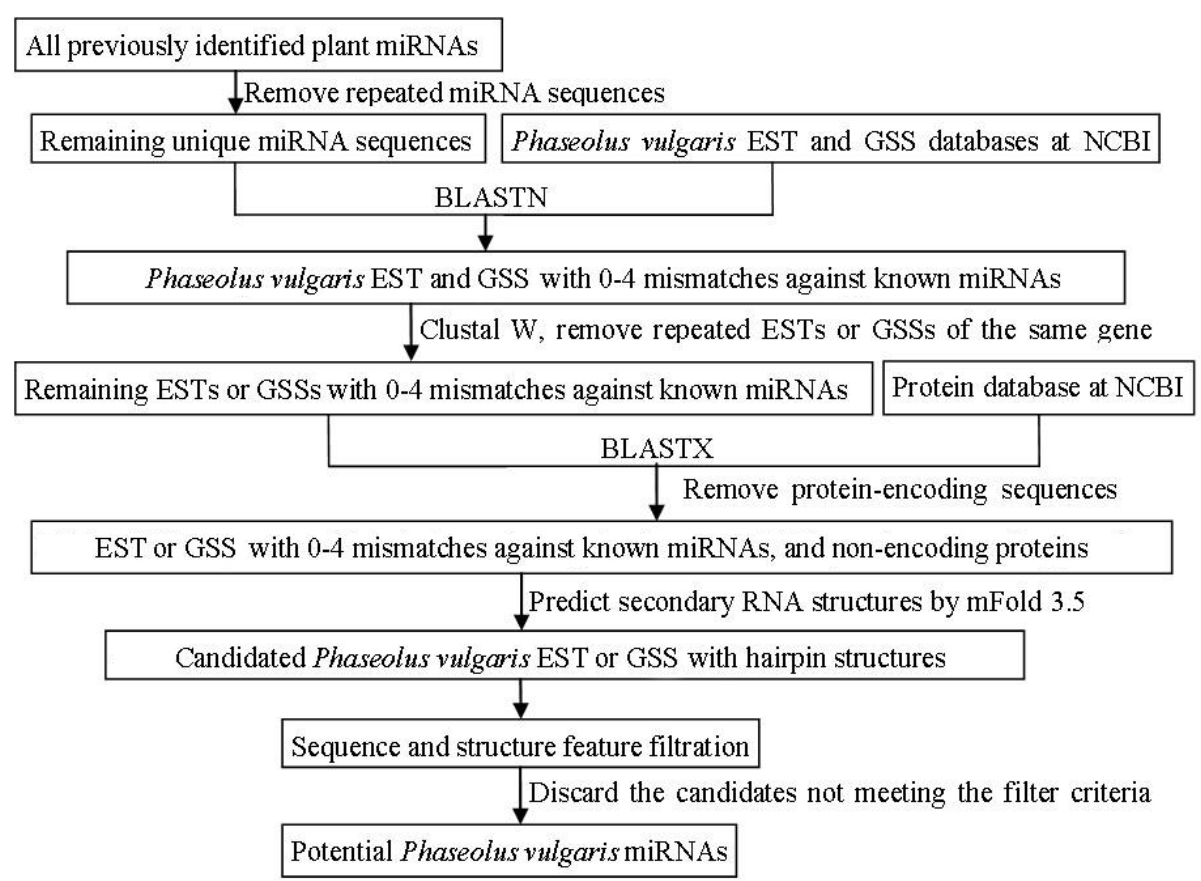

Figure 1. Procedure for the prediction of Phaseolus vulgaris potential miRNAs by searching EST and GSS homologs of previously known plant miRNAs.

\section{Prediction of targets for novel miRNAs}

Previous studies have demonstrated that most known plant miRNAs bind to their targets with perfect or nearly perfect sequence complementarity (Bartel, 2004). This phenomenon provides a powerful approach to identify plant miRNA targets by searching for miRNA homologs. In this study, targets of newly identified miRNAs were predicted by the web tool psRNATarget, using the $P$. vulgaris (Bean) DFCI Gene index (PHVGI) release 3.1 as the sequence library for the target search. The following criteria were used to identify miRNA targets: 1) no more than 4 mismatches were allowed between the mature miRNA and its potential target site; 2) no more than 1 mismatch was allowed at nucleotide positions $1-9 ; 3$ ) no more than 2 consecutive mismatches were allowed; and 4) no mismatches were allowed at positions 10 and 11.

\section{Phylogenetic analysis of the new miRNAs}

Because most plant miRNAs and their precursor sequences are derived from the same gene families, they are strongly conserved and have high sequence identity, even between distantly related species. The evolutionary relationships between the precursor sequences of the 
novel $P$. vulgaris miRNAs and their counterparts in the same family from other plant species were analyzed by MEGA 5.1 (http://www.megasoftware.net/mega.php).

\section{RESULTS AND DISCUSSION}

\section{Identification of potential miRNAs}

The miRNA homology-based EST and GSS blast analyses were conducted by comparing 1804 unique plant miRNAs with the $P$. vulgaris EST and GSS databases. After searching homology sequences, the redundant sequences of the same genes were removed, and then the protein-coding sequences was removed. This filtering, accordance with the screening criteria, left 828 EST and 765 GSS, which were selected as miRNA candidates. The secondary structures of the candidate miRNA sequences were predicted by MFold 3.5, and finally manually inspected according to the screening criteria described in the methods. Finally, a total of 27 potential $P$. vulgaris miRNAs, belonging to 24 miRNA families, were identified. Within the 27 predicted miRNAs, 12 miRNAs were identified from the EST and 15 miRNAs from the GSS (Table 1). Considering the known P. vulgaris miRNAs deposited in the miRNA database, all of the mature miRNA sequences identified in our study were compared with known $P$. vulgaris miRNAs. The results indicated that only 3 miRNAs had identical sequence with known $P$. vulgaris miRNAs, with the other 24 miRNAs being newly identified for this species (Table 1).

\begin{tabular}{|c|c|c|c|c|c|c|c|c|c|c|}
\hline miRNAs & GenBank ID & Source & $\begin{array}{l}\text { miRNA mature sequences } \\
\left(5^{\prime} \rightarrow 3^{\prime}\right)\end{array}$ & Location & NM (nt) & LM (nt) & LP (nt) & $(\mathrm{A}+\mathrm{U}) \%$ & $\begin{array}{c}\text { MFEs } \\
(\mathrm{kcal} / \mathrm{mol})\end{array}$ & MFEIs \\
\hline $\operatorname{miR} 156$ & EI480836 & GSS & uugacagaagagagagagcaca & 5 & 0 & 22 & 85 & 54.12 & -43.60 & 1.12 \\
\hline $\operatorname{miR} 167 \mathrm{a}$ & FE692413 & EST & ugaagcugccagcaugaucua & 5 & 0 & 21 & 86 & 50.00 & -36.90 & 0.86 \\
\hline $\operatorname{miR} 167 b$ & FD792204 & EST & ugaagcugccagcaugaucuga & 5 & 0 & 22 & 80 & 56.25 & -44.70 & 1.28 \\
\hline $\operatorname{miR} 168$ & FE674450 & EST & ucgcuuggugcaggucgggaa & 5 & 0 & 21 & 114 & 42.11 & -60.50 & 0.92 \\
\hline $\operatorname{miR} 169$ & EI430521 & GSS & ugagccaaggauggcuugecg & 5 & 0 & 21 & 86 & 53.49 & -57.60 & 1.44 \\
\hline miR171a & EI500954 & GSS & ugauugagccgcgucaauauc & 3 & 0 & 21 & 98 & 63.27 & -45.80 & 1.27 \\
\hline $\operatorname{miR} 171 b$ & GW908487 & EST & uaauugagccgcgucaauauc & 3 & 1 & 21 & 81 & 58.02 & -38.00 & 1.18 \\
\hline $\operatorname{miR} 172$ & EI491452 & GSS & aagacaucuccaagauucaga & 3 & 4 & 21 & 91 & 67.03 & -30.40 & 1.01 \\
\hline $\operatorname{miR} 397$ & EI458339 & GSS & ucauugagugcagcauugauc & 3 & 2 & 21 & 186 & 59.14 & -70.00 & 0.92 \\
\hline $\operatorname{miR} 398$ & EI460202 & GSS & uguguucucaggucaccccuu & 3 & 0 & 21 & 112 & 60.71 & -58.20 & 1.32 \\
\hline $\operatorname{miR} 399$ & EG594328 & EST & ugccaaaggagaguugeccug & 3 & 0 & 21 & 75 & 54.67 & -38.80 & 1.14 \\
\hline $\operatorname{miR} 403$ & EI488011 & GSS & uuagauucacgcacaaacuug & 3 & 0 & 21 & 97 & 59.79 & -42.16 & 1.08 \\
\hline $\operatorname{miR} 408$ & FE687314 & EST & augcacugccucuucccuggc & 3 & 0 & 21 & 112 & 50.89 & -60.80 & 1.11 \\
\hline $\operatorname{miR} 414$ & EI477854 & GSS & accuuucaucaucaucauca & 5 & 4 & 21 & 150 & 48.67 & -79.30 & 1.03 \\
\hline $\operatorname{miR} 447$ & EI444518 & GSS & uuggggaagagaugauuuguuu & 5 & 3 & 22 & 102 & 67.71 & -31.40 & 0.95 \\
\hline $\operatorname{miR} 1436$ & EI486878 & GSS & acauuaugaaacggagggagu & 3 & 2 & 21 & 169 & 66.27 & -78.10 & 1.37 \\
\hline $\operatorname{miR} 1514$ & FE709266 & EST & uucauuuugaaaauaggcauug & 5 & 0 & 22 & 127 & 66.93 & -45.50 & 1.08 \\
\hline $\operatorname{miR} 1888$ & EI490416 & GSS & caaguuaagaauugagaagaa & 3 & 3 & 21 & 108 & 68.37 & -33.80 & 0.99 \\
\hline $\operatorname{miR} 2118$ & HS104359 & EST & uugccgauuccacccauuccua & 3 & 0 & 22 & 92 & 57.61 & -40.20 & 1.03 \\
\hline $\operatorname{miR} 2595$ & EI433455 & GSS & ucaauuuucuucuugaugucu & 5 & 4 & 21 & 108 & 62.04 & -35.40 & 0.86 \\
\hline $\operatorname{miR} 2628$ & FE898012 & EST & agagaaagaaagaugaguaa & 3 & 4 & 20 & 116 & 61.21 & -40.90 & 0.91 \\
\hline $\operatorname{miR} 2672$ & EI468445 & GSS & uuaaucgaccaaguugguaaaa & 5 & 4 & 22 & 140 & 65.00 & -50.40 & 1.03 \\
\hline $\operatorname{miR} 2919$ & HS103295 & EST & aagggggggggggggaauu & 5 & 3 & 19 & 89 & 30.34 & -71.70 & 1.16 \\
\hline $\operatorname{miR} 5021$ & GW909603 & EST & ugagaagaugaugaggaaau & 5 & 4 & 20 & 202 & 59.90 & -71.00 & 0.88 \\
\hline miR5205 & EI470379 & GSS & uauauauuuggggacggagguagu & 3 & 4 & 24 & 87 & 64.37 & -47.80 & 1.54 \\
\hline $\operatorname{miR} 5658 \mathrm{a}$ & FE698270 & EST & augaugaugaugaugaugaug & 3 & 2 & 21 & 68 & 54.42 & -27.60 & 0.89 \\
\hline $\operatorname{miR} 5658 b$ & EI503792 & GSS & augaugaugaugaugaugucu & 5 & 3 & 21 & 146 & 62.33 & -52.70 & 0.96 \\
\hline
\end{tabular}

The miRNAs newly identified in P. vulgaris are shown in red color. NM = No. of mismatch; LM = length of mature miRNAs; $\mathrm{LP}=$ length of precursors; $\mathrm{MFEs}=$ minimal folding free energies; MFEIs = minimal foldingfree energy indexes. 
The characteristics of the identified P. vulgaris miRNAs were studied to distinguish miRNAs from other small RNAs, such as miRNA mature sequences, length of mature miRNAs, miRNA location, length of precursors, MFEs, and MFEIs (Table 1). The identified $P$. vulgaris pre-miRNAs were between 68 and $202 \mathrm{nt}$ in length, with an average of 111.37 $\pm 34.17 \mathrm{nt}$; however, the majority of the pre-miRNAs (24 of 27 or $88.89 \%$ ) were $60-150$ nt in length (Table 1). These results matched the lengths of most previously predicted premiRNAs in plant species (Zhang et al., 2007; Unver and Budak, 2009; Barozai et al., 2012; Neutelings et al., 2012). Although the identified P. vulgaris miRNA precursors were of a wide range of lengths, they could be folded into the typical miRNA secondary structures (Figure 2). The lengths of these identified mature miRNA sequences ranged from 19 to 24 nucleotides; however, most of the potential miRNAs (17 of 27 or $62.96 \%$ ) were $21 \mathrm{nt}$ in length (Table 1). This length was quite similar to other mature miRNAs already identified in other plants (Zhang et al., 2006a; Xie et al., 2010; Barozai et al., 2012; Neutelings et al., 2012).

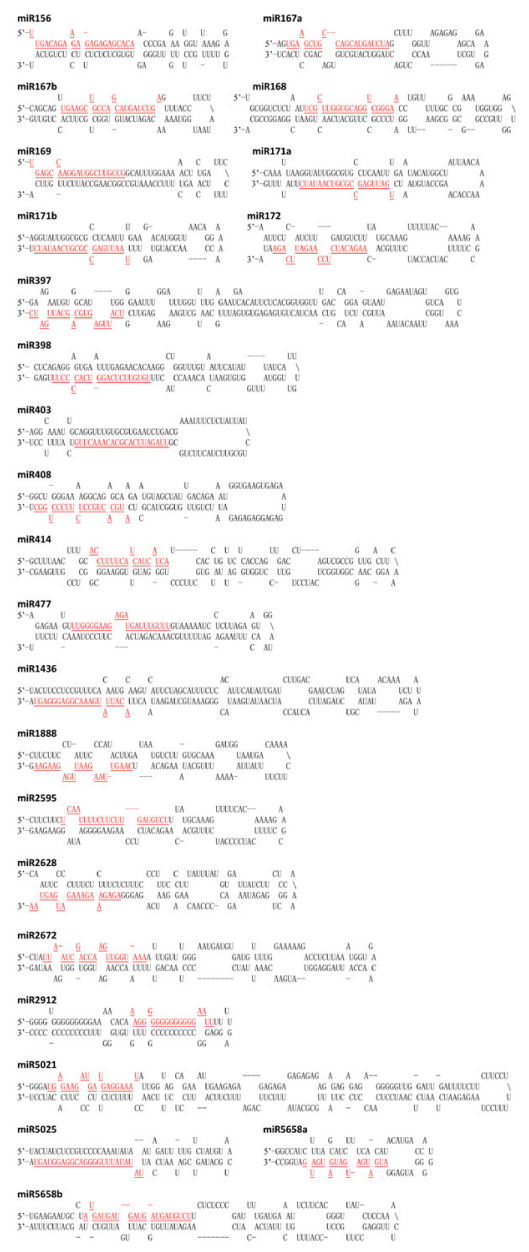

Figure 2. Predicted hairpin secondary structures of the newly identified Phaseolus vulgaris miRNA precursors; mature miRNA sequences are underlined. 
The A+U contents of these predicted P. vulgaris pre-miRNA sequences ranged from 30.34 to $68.37 \%$, with an average of $57.95 \pm 8.53 \%$ (Table 1 ), which closely matched the results of previous studies (Ambros et al., 2003; Neutelings et al., 2012). MFE values are important for evaluating the stability of RNA secondary structures. In general, the lower the MFE, the more stable the secondary structure of an RNA sequence. The MFE values of the identified $P$. vulgaris miRNA precursors varied broadly from $-79.30 \mathrm{kcal} / \mathrm{mol}$ to $-27.60 \mathrm{kcal} / \mathrm{mol}$, with an average of $-49.38 \pm 14.95 \mathrm{kcal} / \mathrm{mol}$ (Table 1 ). The MFEI of each potential miRNA precursor was calculated for the precise discrimination of the miRNA from other types of small RNAs (Adai et al., 2005; Zhang et al., 2005). We found that the precursors of these miRNAs had high MFEIs, ranging from 0.86 to 1.54 (Table 1), with an average of $1.09 \pm 0.18$. These values were significantly higher compared to those reported for tRNAs (0.64), rRNAs (0.59), and mRNAs (0.62-0.66), indicating that these identified P. vulgaris miRNAs are probably true miRNAs (Zhang et al., 2006b).

Mature miRNA sequences have been reported to be evenly located on the 2 arms of the stem-loop hairpin structures of potential pre-miRNAs (Gorodkin et al., 2006). We also obtained the same result in our study for the 27 identified P. vulgaris miRNAs, of which 13 (48.15\%) were found to be located on the $5^{\prime}$-arms of the stem-loop hairpin structures, while the other 14 $(51.85 \%)$ were located on the 3 '-arms (Table 1 and Figure 2).

\section{Targets of newly predicted miRNAs}

The prediction of targets for the identified miRNAs is anticipated to help us elucidate the important function and regulation of these novel miRNAs in P. vulgaris. Most plant miRNAs are perfectly or near-perfectly complementary with their targets (Schwab et al., 2005). For this reason, searching potential miRNA targets by blasting the mature miRNA sequences against the cDNA or EST database is considered a reliable technique. Nevertheless, several studies have demonstrated that many target genes have 1 to 4 nucleotide mismatches with mature miRNA sequences (Xie et al., 2010, 2011).

We identified a total of 92 potential targets with known functions for the 13 newly predicted miRNA families in $P$. vulgaris. The number of potential targets for every miRNA family ranged from 1 (miR398) to 23 (miR5658) (Table 2). However, 18 targets with unknown functions have not been listed here. Because a small number of sequences could be employed for target searching, no targets were found for miR168, miR172, miR403, miR408, miR1436, miR2672, miR2919, or miR5205 in P. vulgaris.

The 92 potential targets belonged to several gene families, and had multiple biological functions. A total of 24 predicted targets for new P. vulgaris miRNAs were transcription factors (Table 2). In addition to the transcription factors, their predicted targeted genes were involved in a broad range of biological processes, such as abiotic stress response, metabolism, transportation, disease resistance, and signal transduction.

Highly conserved $P$. vulgaris miRNAs also had conserved miRNA target sites on specific target genes, which was also observed in previous studies of other plants (Frazier et al., 2010; Xie et al., 2010, 2011). For example, it has been established that the plant-specific transcription factor, squamosa promoter binding protein, is involved in regulating changes during the early flower development and vegetative phase. In addition, it has been widely accepted that this transcription factor is a conserved target of the miR156 family in plants (Yin et al., 2008; Xie et al., 2011). In our study, 2 squamosa promoter binding protein-encoding genes were also identified as the targets of miR156 in P. vulgaris (Table 2). Some CCAAT- 
Table 2. Predicted targets of the newly identified miRNAs in Phaseolus vulgaris.

\begin{tabular}{|c|c|c|c|}
\hline miRNA families & Targeted protein & Functions of targetes & Targeted genes \\
\hline \multirow[t]{4}{*}{$\operatorname{miR} 156$} & Squamosa promoter binding protein & Transcription factor & FE706771 (1) TC26626 (1) \\
\hline & $\mathrm{ABC}$ transporter $\mathrm{E}$ family member & Transporter & TC23933 (2) \\
\hline & Aquaporin SIP $1 ; 2$ & & TC22849 (2.5) \\
\hline & Haloalkane dehalogenase 2-like & Metabolism & TC20482(3) \\
\hline \multirow[t]{2}{*}{$\operatorname{miR} 167$} & Dioxygenase & Metabolism & TC20369 (2.5) TC27684 (2.5) \\
\hline & Acyltransferase & Metabolism & TC26796 (3) \\
\hline \multirow[t]{2}{*}{$\operatorname{miR} 169$} & CCAAT-binding transcription factor & Transcription factor & $\begin{array}{l}\text { TC20287 (1) FE700223 (1.5) } \\
\text { TC28312 (2)TC25936 (2) }\end{array}$ \\
\hline & Auxin Efflux Carrier & Signal transduction & CV537472 (3) \\
\hline \multirow[t]{3}{*}{$\operatorname{miR} 171$} & GRAS family transcription factor & Transcription factor & TC20655 (0.5)TC22093 (0.5) \\
\hline & ATP-dependent chaperone ClpB & & TC28957 (3) \\
\hline & Ac transposase-like protein & & FD794302 (2.5) \\
\hline \multirow[t]{3}{*}{ miR397 } & Laccase-17-like & Metabolism & CB541070 (0.5) \\
\hline & Lipoxygenase & Metabolism & TC28581 (2.5) \\
\hline & Glucosyltransferase & Metabolism & TC20381 (3) \\
\hline \multirow[t]{2}{*}{$\operatorname{miR} 398$} & Monogalactosyldiacylglycerol synthase & Metabolism & TC20478 (3) \\
\hline & Pollen-specific protein SF3 & Transcription factor & TC26066 (2) \\
\hline \multirow[t]{14}{*}{$\operatorname{miR} 414$} & Adipocyte plasma membrane protein & Signal transduction & TC20889 (1.5) \\
\hline & Tubulin & & FE691368 (2) \\
\hline & TOC1 & Transcription factor & TC21380 (2.5) \\
\hline & Zinc finger family protein & Transcription factor & TC26868 (2.5) TC20427 (3) FE691610 (3) \\
\hline & Histidine acid phosphatase & Metabolism & TC25565 (2.5) \\
\hline & $\mathrm{ABC}$ transporter & Transporter & TC23456 (2.5) \\
\hline & Transcription factor 25 -like & Transcription factor & TC31171 (2.5) \\
\hline & F-box/kelch-repeat protein & Transcription factor & CB540056 (3) \\
\hline & HMG1/2-like protein & Transcription factor & CV543900 (3) \\
\hline & Ethylene-responsive late embryogenesis & Stress response & TC30955 (3) \\
\hline & Transcription initiation factor TFIID & Transcription factor & CV541321 (3) \\
\hline & Cell number regulator 5-like & Development & CV543778 (3) \\
\hline & Ribosomal protein S6 family protein & & CV534067 (3) \\
\hline & bZIP transcription factor & Transcription factor & TC31739 (3) \\
\hline \multirow[t]{4}{*}{$\operatorname{miR} 447$} & Tubulin A & & TC20420 (3)TC21705 (3) TC31555 (3) \\
\hline & Xyloglucan endotransglucosylase & Metabolism & TC31914 (3) \\
\hline & Rab family protein & & TC26265 (3) \\
\hline & ATP-dependent protease La & Metabolism & FD792124 (3) \\
\hline \multirow[t]{3}{*}{$\operatorname{miR} 1888$} & U-box domain-containing protein & & $\mathrm{TC} 24440(2)$ \\
\hline & AT hook motif DNA-binding family & Transcription factor & FD790511 (3) \\
\hline & Glycosyltransferase & Metabolism & TC25804 (3) \\
\hline \multirow[t]{6}{*}{$\operatorname{miR} 2595$} & GATA transcription factor MED5 & Transcription factor & $\mathrm{TC} 27879(2)$ \\
\hline & NDPH:quinone oxidoreductase & Metabolism & TC23497 (2.5) \\
\hline & SURP motif-containing protein & & TC29671 (3) \\
\hline & Heat shock protein 70 & Stress response & TC31270 (3) \\
\hline & $40 \mathrm{~S}$ ribosomal protein S5 & & TC23413 (3) \\
\hline & Transcription factor MYB86 & Transcription factor & CV540785 (3) \\
\hline \multirow[t]{10}{*}{$\operatorname{miR} 2628$} & Jasmonate Zim-domain protein & Signal transduction & $\mathrm{TC} 21766(2)$ \\
\hline & Anion-selective channel protein & Signal transduction & TC24615 (2) \\
\hline & Lipase class 3 family protein & Metabolism & TC29044 (2.5) \\
\hline & F-box protein PP2-A13-like & Transcription factor & TC25861 (2.5) \\
\hline & ATP-dependent RNA helicase eIF4A & & TC25757 (2.5) \\
\hline & WD repeat-containing protein & Signal transduction & TC28514 (2.5) \\
\hline & Gibberellin 20-oxidase & Signal transduction & TC21749 (3) \\
\hline & Developmental protein FluG & Development & FE690630 (3) \\
\hline & Leucine zipper protein ATHB-6 & Transcription factor & TC22980 (3) \\
\hline & MADS-box protein & Transcription factor & FE898584 (3) \\
\hline \multirow[t]{5}{*}{$\operatorname{miR} 5021$} & NOD3 & Development & FG231724 (2.5) \\
\hline & SPX domain-containing protein 3 & & TC27882 (2.5) \\
\hline & SufE-like protein & Metabolism & TC21780 (3) \\
\hline & Aryl-alcohol dehydrogenase & Metabolism & TC21619(3) \\
\hline & Uridine-cytidine kinase C-like & Metabolism & FE711956 (3) TC23026 (3) \\
\hline
\end{tabular}

Continued on next page 


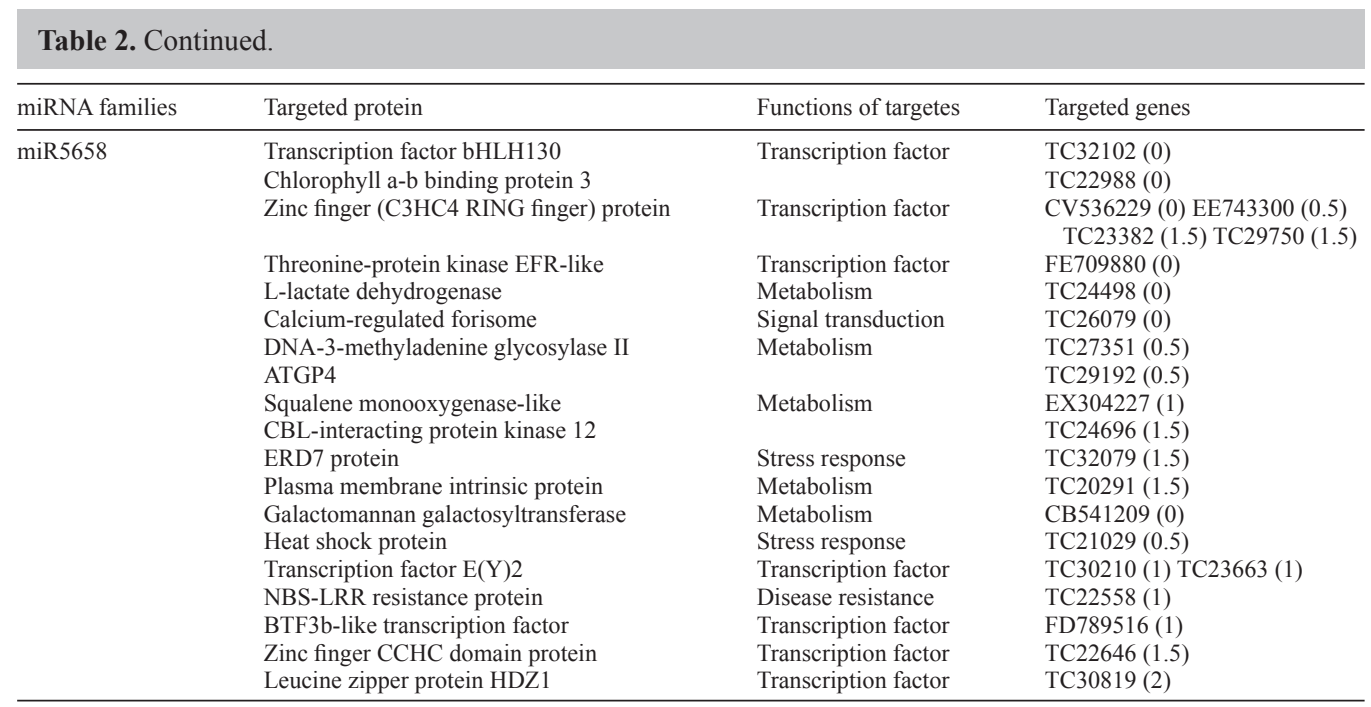

box binding transcription factors have been reported as targets of the ath-miR169 family in Arabidopsis (Xie et al., 2005). MtHAP2-1 is a new transcription factor of the CCAAT-binding family, and has been reported to be regulated by miR169 in Medicago truncatula, with miR169 being considered essential for nodule development in M. truncatula (Combier et al., 2006). We also found 4 CCAAT-binding transcription factor encoding targets for the miR169 family in $P$. vulgaris (Table 2). Recent research revealed that the miR171 family negatively regulates shoot branching and decreases primary root elongation by targeting GRAS gene family members SCARECROW-LIKE6-II (SCL6-II), SCL6-III, and SCL6-IV for cleavage in Arabidopsis (Wang et al., 2010). Interestingly, MtNsp2, which is targeted by the miR171 family in M. truncatula, encodes a GRAS transcription factor essential for nodule symbiosis signaling (Branscheid et al., 2011). Here, 2 GRAS transcription factors were predicted to be targeted by miR171 in $P$. vulgaris (Table2). Previous studies showed that several AUXIN RESPONSE FACTORS (ARFS) were targets for miR167 family in Oryza sativa (Liu et al., 2012). These ARFs play important roles in plant growth and development, by regulating the expression of auxin-responsive genes. Copper homeostasis is regulated by conserved miR398 in Arabidopsis, whereby miR398 directs the degradation of Copper/zinc Superoxide Dismutase mRNA when copper is limited (Yamasaki et al., 2007). Nevertheless, these conserved targets were not identified for the miR167 and miR398 families in P. vulgaris, mainly because the sequence information was incomplete.

The general transcription factor TFIID contains the TATA-binding protein (TBP) and a set of 13-14 TBP-associated factors (TAFs). TFIID is recruited by the activity of promoters, and possibly repressed genes (Cler et al., 2009). We predicted that miR414 directed the regulation of TFIID in P. vulgaris. Several other transcription factor groups were also detected as the targets of miR414 (Table 2), such as, bZIP transcription factor, which is involved in plant development and stress responses (Kaminaka et al., 2006). F-box/kelch-repeat protein controls the daytime expression pattern of the CONSTANS (CO) protein, and serves as an important regulator of plant flowering (Imaizumi et al., 2005). TIMING OF CAB EXPRESSION 1 (TOC1) is the critical clock component, and is a transcriptional regulator that binds DNA. TOC1 controls growth and development in Arabidopsis (Gendron et al., 2012). 
MYB domain transcription factors play key regulatory roles in plant developmental processes, metabolism, and responses to biotic and abiotic stress response (Dubos et al., 2010). MYB transcription factors have been proven to be the targets of miR414 in potato and the model grass species Brachypodium distachyon (Unver and Budak, 2009; Xie et al., 2011). In contrast, in this study, transcription factor MYB86 was predicted to be the target of miR2595 in P. vulgaris. miR414 and miR5658 targeted zinc finger transcription factors are a superfamily of proteins involved in numerous activities of plant growth and development, and are also known to regulate resistance mechanisms for various biotic and abiotic stresses (Giri et al., 2011). The basic/helixloop-helix (bHLH) transcription factors that control cell proliferation and cell lineage establishment are potential targets for P. vulgaris miR5658. miR2628 and miR5658 target the mRNA of homeobox-leucine zipper transcription factor, which is reported to regulate leaf polarity and vascular differentiation via the miR165/166 family (Jung and Park, 2007). Previous studies have revealed that miR444 family members target MADS-box transcription factors that are involved in diverse biological functions, including tolerance to abiotic stresses (Ding et al., 2011). Yet, in this study, MADS-box transcription factor was identified as the target of $P$. vulgaris miR2628.

Some of the newly identified $P$. vulgaris miRNAs also targeted genes involved in metabolism (Table 2), such as dioxygenase, acyltransferase, laccase, lipoxygenase, glucosyltransferase, monogalactosyldiacylglycerol synthase, L-lactate dehydrogenase, lipase, squalene monooxygenase, and galactomannan galactosyltransferase. We also found that several novel $P$. vulgaris miRNAs have complementary sites with the jasmonate zim-domain proteins, anion-selective channel protein, WD repeat-containing protein, gibberellin 20-oxidase, and calcium-regulated forisome (Table 2). Therefore, all of these genes might play important roles in signal transduction.

Many studies have shown that plant miRNAs are involved in biotic and abiotic stress (Xie et al., 2010; Gao et al., 2011; Sunkar et al., 2012). We also identified the stress-related proteins targeted by $P$. vulgaris miRNAs. For instance, miR2595 and miR5658 also targeted the heat shock proteins that are expressed in response to heat stress, which indicates the important role of these miRNAs during heat stress. Most plant resistance genes (R genes) encode nucleotidebinding site leucine-rich repeat (NBS-LRR) proteins (McHale et al., 2006). The NBS-LRR resistance protein was predicted to be the target of the P. vulgaris miR5658 family.

\section{Phylogenetic analysis of the new miRNAs}

Mature miRNA sequences, along with their corresponding precursor sequences, are highly conserved among different plant species (Zhang et al., 2006a). This phenomenon provides opportunities for the investigation of evolutionary relationships of miRNAs belonging to the same families in different plant species. To gain further insights about the evolutionary relationships of the newly identified miRNAs and their counterparts in other plant species, the phylogenetic relationships of several conserved $P$. vulgaris miRNAs with other members from the same families were analyzed by MEGA 5.1, including the miR156, miR167, miR168, miR169, miR171, miR398, and miR408 family. The precursor sequences of these miRNA families were obtained from the miRNA database (Release August 19, 2012, http://www.mirbase.org/).

Phylogenetic trees for 7 conserved miRNA families were constructed based on the premiRNA sequence comparisons. We concluded from the phylogenetic trees that the evolutionary relationships of $P$. vulgaris miRNAs with their counterparts from diverse plant species differed in different families. For instance, most members of the miR168 and miR408 families from legume plants were clustered into 1 group, indicating that these two families are highly conserved 
in legume plants (Figure 3A and B). In comparison, the members of the miR156, miR167,

A

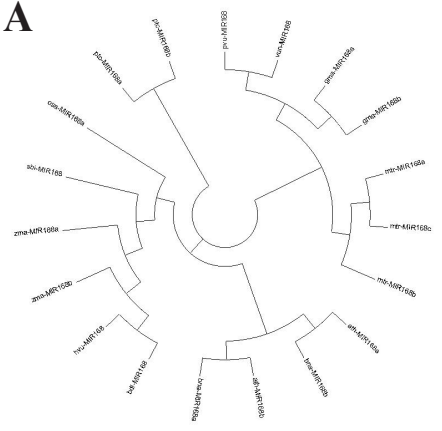

C

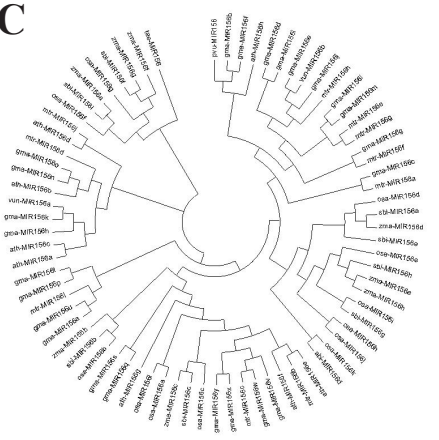

$\mathbf{E}$

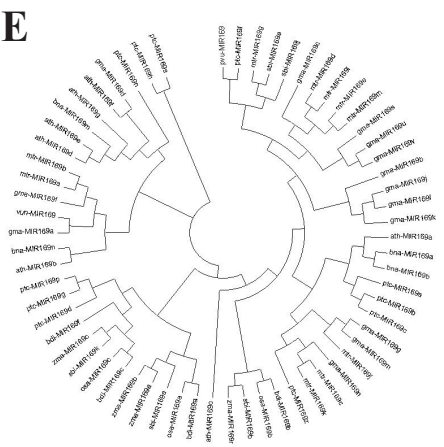

B

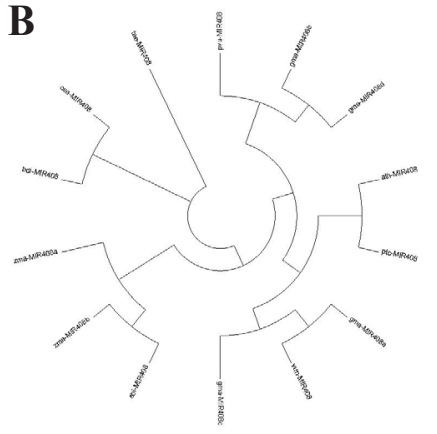

D

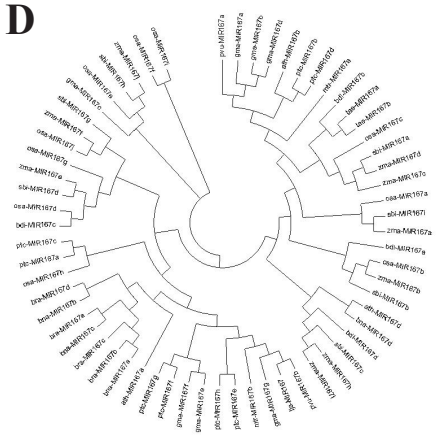

$\mathbf{F}$

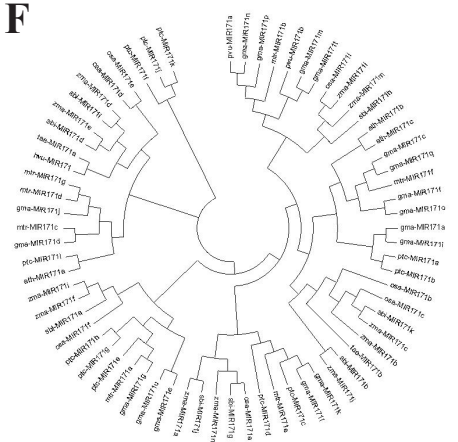

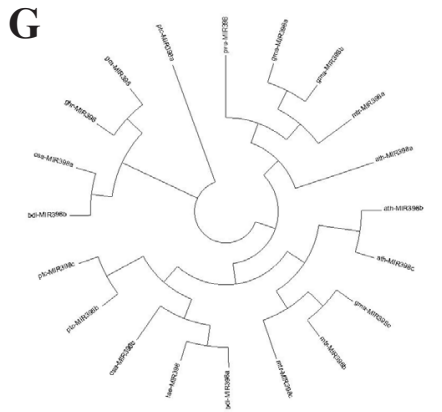

Figure 3. Phylogenetic trees obtained by aligning the precursor sequences of miRNAs in different families. A. MIR168 family; B. MIR408 family; C. MIR156 family; D. MIR167 family; E. MIR169 family; F. MIR171 family; G. MIR398 family. 
miR169, miR171, and miR398 families from legume plants were clustered into more than 1 group, indicating that these miRNA families might have quickly diverged in legume plants. However, all of the $P$. vulgaris miRNAs from these families had some counterparts from legume plants on the same branches. For instance, pvu-MIR156 was the most closely related to gma-MIR156b and gma-MIR156f in the plant miR156 family (Figure 3C). In comparison, pvu-MIR167, gmaMIR167a, gma-MIR167b, gma-MIR167d, and mtr-MIR167a were clustered into 1 small branch (Figure 3D). pvu-MIR169 and mtr-MIR169g were located on 1 small branch (Figure 3E). pvuMIR171a, pvu-MIR171b, gma-MIR171m, gma-MIR171n, gma-MIR171p, gma-MIR171t, and mtr-MIR171b formed a small group (Figure 3F). pvu-MIR398, gma-MIR398a, gma-MIR398b, and mtr-MIR398a formed a cluster on another small branch (Figure 3G). In addition, 2 members of the $P$. vulgaris miR171 family were closely related on the phylogenetic tree. Overall, these results indicate that different miRNAs might evolve at different rates in the plant kingdom, with some plant miRNA families being more conserved than the others.

\section{ACKNOWLEDGMENTS}

Research supported by the Scientific Research Project of Beijing Educational Committee, China (\#PXM2011_014207_000037), and the National Natural Science Foundation of China (\#31301312).

\section{REFERENCES}

Adai A, Johnson C, Mlotshwa S, Archer-Evans S, et al. (2005). Computational prediction of miRNAs in Arabidopsis thaliana. Genome Res. 15: 78-91.

Ambros V, Bartel B, Bartel DP, Burge CB, et al. (2003). A uniform system for microRNA annotation. RNA 9: 277-279.

Barozai MY, Baloch IA and Din M (2012). Identification of MicroRNAs and their targets in Helianthus. Mol. Biol. Rep. 39: 2523-2532.

Bartel DP (2004). MicroRNAs: genomics, biogenesis, mechanism, and function. Cell 116: 281-297.

Branscheid A, Devers EA, May P and Krajinski F (2011). Distribution pattern of small RNA and degradome reads provides information on miRNA gene structure and regulation. Plant Signal. Behav. 6: 1609-1611.

Broughton WJ, Hernández G, Blair M and Beebe S (2003). Beans (Phaseolus spp.) - model food legumes. Plant Soil 252: 55-128.

Cler E, Papai G, Schultz P and Davidson I (2009). Recent advances in understanding the structure and function of general transcription factor TFIID. Cell Mol. Life Sci. 66: 2123-2134.

Combier JP, Frugier F, de Billy F, Boualem A, et al. (2006). MtHAP2-1 is a key transcriptional regulator of symbiotic nodule development regulated by microRNA169 in Medicago truncatula. Genes Dev. 20: 3084-3088.

Dai X and Zhao PX (2011). psRNATarget: a plant small RNA target analysis server. Nucleic Acids Res. 39: W155-W159.

Ding Y, Chen Z and Zhu C (2011). Microarray-based analysis of cadmium-responsive microRNAs in rice (Oryza sativa). J. Exp. Bot. 62: 3563-3573.

Dubos C, Stracke R, Grotewold E, Weisshaar B, et al. (2010). MYB transcription factors in Arabidopsis. Trends Plant Sci. 15: 573-581.

Frazier TP, Xie F, Freistaedter A, Burklew CE, et al. (2010). Identification and characterization of microRNAs and their target genes in tobacco (Nicotiana tabacum). Planta 232: 1289-1308.

Gao P, Bai X, Yang L, Lv D, et al. (2011). osa-MIR393: a salinity- and alkaline stress-related microRNA gene. Mol. Biol. Rep. 38: 237-242.

Gendron JM, Pruneda-Paz JL, Doherty CJ, Gross AM, et al. (2012). Arabidopsis circadian clock protein, TOC1, is a DNAbinding transcription factor. Proc. Natl. Acad. Sci. U. S. A. 109: 3167-3172.

Giri J, Vij S, Dansana PK and Tyagi AK (2011). Rice A20/AN1 zinc-finger containing stress-associated proteins (SAP1/11) and a receptor-like cytoplasmic kinase (OsRLCK253) interact via A20 zinc-finger and confer abiotic stress tolerance in transgenic Arabidopsis plants. New Phytol. 191: 721-732.

Gorodkin J, Havgaard JH, Enstero M, Sawera M, et al. (2006). MicroRNA sequence motifs reveal asymmetry between the 
stem arms. Comput. Biol. Chem. 30: 249-254.

Imaizumi T, Schultz TF, Harmon FG, Ho LA, et al. (2005). FKF1 F-box protein mediates cyclic degradation of a repressor of CONSTANS in Arabidopsis. Science 309: 293-297.

Jung JH and Park CM (2007). MIR166/165 genes exhibit dynamic expression patterns in regulating shoot apical meristem and floral development in Arabidopsis. Planta 225: 1327-1338.

Kaminaka H, Näke C, Epple P, Dittgen J, et al. (2006). bZIP10-LSD1 antagonism modulates basal defense and cell death in Arabidopsis following infection. EMBO J. 25: 4400-4411.

Larue CT, Wen J and Walker JC (2009). A microRNA-transcription factor module regulates lateral organ size and patterning in Arabidopsis. Plant J. 58: 450-463.

Li F, Pignatta D, Bendix C, Brunkard JO, et al. (2012). MicroRNA regulation of plant innate immune receptors. Proc. Natl. Acad. Sci. U. S. A. 109: 1790-1795.

Liu H, Jia SH, Shen DF, Liu J, et al. (2012). Four AUXIN RESPONSE FACTOR genes downregulated by microRNA167 are associated with growth and development in Oryza sativa. Funct. Plant Biol. 39: 744.

Mallory AC, Bartel DP and Bartel B (2005). MicroRNA-directed regulation of Arabidopsis AUXIN RESPONSE FACTOR17 is essential for proper development and modulates expression of early auxin response genes. Plant Cell 17: $1360-1375$.

McHale L, Tan X, Koehl P and Michelmore RW (2006). Plant NBS-LRR proteins: adaptable guards. Genome Biol. 7: 212. Megraw M and Hatzigeorgiou AG (2010). MicroRNA promoter analysis. Methods Mol. Biol. 592: 149-161.

Neutelings G, Fenart S, Lucau-Danila A and Hawkins S (2012). Identification and characterization of miRNAs and their potential targets in flax. J. Plant Physiol. 169: 1754-1766.

Schwab R, Palatnik JF, Riester M, Schommer C, et al. (2005). Specific effects of microRNAs on the plant transcriptome. Dev. Cell 8: 517-527.

Sunkar R, Li YF and Jagadeeswaran G (2012). Functions of microRNAs in plant stress responses. Trends Plant Sci. 17: 196-203.

Unver T and Budak H (2009). Conserved microRNAs and their targets in model grass species Brachypodium distachyon. Planta 230: 659-669.

Wang L, Mai YX, Zhang YC, Luo Q, et al. (2010). MicroRNA171c-targeted SCL6-II, SCL6-III, and SCL6-IV genes regulate shoot branching in Arabidopsis. Mol. Plant 3: 794-806.

Xie F, Frazier TP and Zhang B (2010). Identification and characterization of microRNAs and their targets in the bioenergy plant switchgrass (Panicum virgatum). Planta 232: 417-434.

Xie F, Frazier TP and Zhang B (2011). Identification, characterization and expression analysis of MicroRNAs and their targets in the potato (Solanum tuberosum). Gene 473: 8-22.

Xie Z, Allen E, Fahlgren N, Calamar A, et al. (2005). Expression of Arabidopsis MIRNA genes. Plant Physiol. 138: $2145-2154$.

Yamasaki H, Abdel-Ghany SE, Cohu CM, Kobayashi Y, et al. (2007). Regulation of copper homeostasis by micro-RNA in Arabidopsis. J. Biol. Chem. 282: 16369-16378.

Yin Z, Li C, Han X and Shen F (2008). Identification of conserved microRNAs and their target genes in tomato (Lycopersicon esculentum). Gene 414: 60-66.

Zhang B, Pan X, Cannon CH, Cobb GP, et al. (2006a). Conservation and divergence of plant microRNA genes. Plant J. 46: 243-259.

Zhang B, Pan X, Cobb GP and Anderson TA (2006b). Plant microRNA: a small regulatory molecule with big impact. Dev. Biol. 289: 3-16.

Zhang B, Wang Q, Wang K, Pan X, et al. (2007). Identification of cotton microRNAs and their targets. Gene 397: 26-37.

Zhang BH, Pan XP, Wang QL, Cobb GP, et al. (2005). Identification and characterization of new plant microRNAs using EST analysis. Cell Res. 15: 336-360.

Zuker M (2003). MFold web server for nucleic acid folding and hybridization prediction. Nucleic Acids Res. 31: $3406-$ 3415. 